\title{
MINIMUM DOMINATING RANDIC ENERGY OF A GRAPH
}

\section{P. S. K. Reddy, K. N. Prakasha, V. M. Siddalingaswamy}

\begin{abstract}
In this paper, we introduce the minimum dominating Randic energy of a graph and computed the minimum dominating Randic energy of graph. Also, obtained upper and lower bounds for the minimum dominating Randic energy of a graph.
\end{abstract}

Mathematics Subject Classification (2010): 05C50.

Key words: minimum dominating set, minimum dominating Randic eigenvalues, minimum dominating Randic energy.

\section{Introduction}

Let $G$ be a simple, finite, undirected graph, The energy $E(G)$ is defined as the sum of the absolute values of the eigenvalues of its adjacency matrix. For more details on energy of graphs (see $[5,6]$ ).

The Randic matrix $R(G)=\left(R_{i j}\right)_{n \times n}$ is given by Bozkurt et al. [1-3].

$$
R_{i j}= \begin{cases}\frac{1}{\sqrt{d_{i} d_{i}}}, & \text { if } v_{i} \sim v_{j}, \\ 0, & \text { otherwise. }\end{cases}
$$

We can see lower and upper bounds on Randic energy in $[1,2,4]$. Some sharp upper bounds for Randic energy of graphs were obtain in [3].

\section{The Minimum Dominating Randic Energy of Graph}

Let $G$ be a simple graph of order $n$ with vertex set $V=\left\{v_{1}, v_{2}, v_{3}, \ldots, v_{n}\right\}$ and edge set $E$. A subset $D$ of $V=V(G)$ is called a dominating set if every vertex in $V-D$ adjacent to a vertex of $D$. Minimum dominating set is called a dominating set of minimum power. Let $D$ be a minimum dominating set of a graph $G$. The minimum dominating Randic matrix $R^{D}(G)=\left(R_{i j}^{D}\right)_{n \times n}$ is given by

$$
R_{i j}^{D}= \begin{cases}\frac{1}{\sqrt{d_{i} d_{i}}}, & \text { if } v_{i} \sim v_{j}, \\ 1, & \text { if } i=j \text { and } v_{i} \in D \\ 0, & \text { otherwise. }\end{cases}
$$

The characteristic polynomial of $R^{D}(G)$ is denoted by $\phi_{R}^{D}(G, \lambda)=\operatorname{det}\left(\lambda I-R^{D}(G)\right)$. Since the minimum dominating Randic Matrix is real and symmetric, its eigenvalues are

(c) 2017 Reddy P. S. K., Prakasha K. N., Siddalingaswamy V. M. 
real numbers and we label them in non-increasing order $\lambda_{1}>\lambda_{2}>\ldots \lambda_{n}$. The minimum dominating Randic Energy is given by

$$
R E_{D}(G)=\sum_{i=1}^{n}\left|\lambda_{i}\right|
$$

Definition 2.1. The spectrum of a graph $G$ is the list of distinct eigenvalues $\lambda_{1}>\lambda_{2}>$ $\cdots>\lambda_{r}$, with their multiplicities $m_{1}, m_{2}, \ldots, m_{r}$, and we write it as

$$
\operatorname{Spec}(\mathrm{G})=\left(\begin{array}{cccc}
\lambda_{1} & \lambda_{2} & \ldots & \lambda_{r} \\
m_{1} & m_{2} & \cdots & m_{r}
\end{array}\right) .
$$

This paper is organized as follows. In the Section 3, we get some basic properties of minimum dominating Randic energy of a graph. In the Section 4, minimum dominating Randic energy of some standard graphs are obtained.

\section{Some Basic Properties of Minimum Dominating Randic Energy of a Graph}

Let us consider

$$
P=\sum_{i<j} \frac{1}{d_{i} d_{j}}
$$

Where $d_{i} d_{j}$ is the product of degrees of two vertices which are adjacent.

Proposition 3.1. The first three coefficients of $\phi_{R}^{D}(G, \lambda)$ are given as follows:

(i) $a_{0}=1$,

(ii) $a_{1}=-|D|$,

(iii) $a_{2}=|D| C_{2}-P$.

$\triangleleft$ (i) From the definition $\Phi_{R}^{D}(G, \lambda)=\operatorname{det}\left[\lambda I-R^{D}(G)\right]$, we get $a_{0}=1$.

(ii) The sum of determinants of all $1 \times 1$ principal submatrices of $R^{D}(G)$ is equal to the trace of $R^{D}(G)$.

$\Rightarrow a_{1}=(-1)^{1}$ trace of $\left[R^{D}(G)\right]=-|D|$.

(iii)

$$
\begin{array}{r}
(-1)^{2} a_{2}=\sum_{1 \leqslant i<j \leqslant n}\left|\begin{array}{ll}
a_{i i} & a_{i j} \\
a_{j i} & a_{j j}
\end{array}\right|=\sum_{1 \leqslant i<j \leqslant n} a_{i i} a_{j j}-a_{j i} a_{i j} \\
=\sum_{1 \leqslant i<j \leqslant n} a_{i i} a_{j j}-\sum_{1 \leqslant i<j \leqslant n} a_{j i} a_{i j}=|D| C_{2}-P . \triangleright
\end{array}
$$

Proposition 3.2. If $\lambda_{1}, \lambda_{2}, \ldots, \lambda_{n}$ are the minimum dominating Randic eigenvalues of $R^{D}(G)$, then

$$
\sum_{i=1}^{n} \lambda_{i}^{2}=|D|+2 P
$$

$\triangleleft$ We know that

$$
\sum_{i=1}^{n} \lambda_{i}^{2}=\sum_{i=1}^{n} \sum_{j=1}^{n} a_{i j} a_{j i}=2 \sum_{i<j}\left(a_{i j}\right)^{2}+\sum_{i=1}^{n}\left(a_{i i}\right)^{2}=2 \sum_{i<j}\left(a_{i j}\right)^{2}+|D|=|D|+2 P . \triangleright
$$


Theorem 3.1. Let $G$ be a graph with $n$ vertices and then

$$
R E^{D}(G) \leqslant \sqrt{n(|D|+2[P])}
$$

where

$$
P=\sum_{i<j} \frac{1}{d_{i} d_{j}}
$$

for which $d_{i} d_{j}$ is the product of degrees of two vertices which are adjacent.

$\triangleleft$ Let $\lambda_{1}, \lambda_{2}, \ldots, \lambda_{n}$ be the eigenvalues of $R^{D}(G)$. Now by Cauchy-Schwartz inequality we have

$$
\left(\sum_{i=1}^{n} a_{i} b_{i}\right)^{2} \leqslant\left(\sum_{i=1}^{n}{a_{i}}^{2}\right)\left(\sum_{i=1}^{n} b_{i}{ }^{2}\right)
$$

Let $a_{i}=1, b_{i}=\left|\lambda_{i}\right|$. Then

$$
\begin{gathered}
\left(\sum_{i=1}^{n}\left|\lambda_{i}\right|\right)^{2} \leqslant\left(\sum_{i=1}^{n} 1\right)\left(\sum_{i=1}^{n}\left|\lambda_{i}\right|^{2}\right), \\
{\left[R E^{D}\right]^{2} \leqslant n(|D|+2 P),} \\
{\left[R E^{D}\right] \leqslant \sqrt{n(|D|+2 P)},}
\end{gathered}
$$

which is upper bound. $\triangleright$

Theorem 3.2. Let $G$ be a graph with $n$ vertices. If $R=\operatorname{det} R^{D}(G)$, then

$$
R E^{D}(G) \geqslant \sqrt{(|D|+2 P)+n(n-1) R^{\frac{2}{n}}}
$$

$\triangleleft$ By definition,

$$
\left(R E^{D}(G)\right)^{2}=\left(\sum_{i=1}^{n}\left|\lambda_{i}\right|\right)^{2}=\sum_{i=1}^{n}\left|\lambda_{i}\right| \sum_{j=1}^{n}\left|\lambda_{j}\right|=\left(\sum_{i=1}^{n}\left|\lambda_{i}\right|^{2}\right)+\sum_{i \neq j}\left|\lambda_{i} \| \lambda_{j}\right| .
$$

Using arithmetic mean and geometric mean inequality, we have

$$
\frac{1}{n(n-1)} \sum_{i \neq j}\left|\lambda_{i} \| \lambda_{j}\right| \geqslant\left(\prod_{i \neq j}\left|\lambda_{i} \| \lambda_{j}\right|\right)^{\frac{1}{n(n-1)}}
$$

Therefore,

$$
\begin{aligned}
{\left[R E^{D}(G)\right]^{2} } & \geqslant \sum_{i=1}^{n}\left|\lambda_{i}\right|^{2}+n(n-1)\left(\prod_{i \neq j}\left|\lambda_{i}\right|\left|\lambda_{j}\right|\right)^{\frac{1}{n(n-1)}} \\
& \geqslant \sum_{i=1}^{n}\left|\lambda_{i}\right|^{2}+n(n-1)\left(\prod_{i=1}^{n}\left|\lambda_{i}\right|^{2(n-1)}\right)^{\frac{1}{n(n-1)}} \\
& =\sum_{i=1}^{n}\left|\lambda_{i}\right|^{2}+n(n-1) R^{\frac{2}{n}}=(|D|+2 P)+n(n-1) R^{\frac{2}{n}}
\end{aligned}
$$


Thus,

$$
R E^{D}(G) \geqslant \sqrt{(|D|+2 P)+n(n-1) R^{\frac{2}{n}}} . \triangleright
$$

\section{Minimum Dominating Randic Energy of Some Standard Graphs}

Theorem 4.1. The minimum dominating Randic energy of a complete graph $K_{n}$ is $R E^{D}\left(K_{n}\right)=\frac{3 n-5}{n-1}$.

$\triangleleft$ Let $K_{n}$ be the complete graph with vertex set $V=\left\{v_{1}, v_{2}, \ldots, v_{n}\right\}$. The minimum dominating set $=D=\left\{v_{1}\right\}$. The minimum dominating Randic matrix is

$$
R^{D}\left(K_{n}\right)=\left[\begin{array}{cccccc}
1 & \frac{1}{n-1} & \frac{1}{n-1} & \cdots & \frac{1}{n-1} & \frac{1}{n-1} \\
\frac{1}{n-1} & 0 & \frac{1}{n-1} & \cdots & \frac{1}{n-1} & \frac{1}{n-1} \\
\frac{1}{n-1} & \frac{1}{n-1} & 0 & \cdots & \frac{1}{n-1} & \frac{1}{n-1} \\
\vdots & \vdots & \vdots & \ddots & \vdots & \vdots \\
\frac{1}{n-1} & \frac{1}{n-1} & \cdots & \frac{1}{n-1} & 0 & \frac{1}{n-1} \\
\frac{1}{n-1} & \frac{1}{n-1} & \cdots & \frac{1}{n-1} & \frac{1}{n-1} & 0
\end{array}\right] .
$$

Characteristic equation is

$$
\left(\lambda+\frac{1}{n-1}\right)^{n-2}\left(\lambda^{2}-\frac{2 n-3}{n-1} \lambda+\frac{n-3}{n-1}\right)=0
$$

and the spectrum is

$$
\operatorname{Spec}_{R}^{D}\left(K_{n}\right)=\left(\begin{array}{ccc}
\frac{(2 n-3)+\sqrt{4 n-3}}{2(n-1)} & \frac{(2 n-3)-\sqrt{4 n-3}}{2(n-1)} & \frac{-1}{n-1} \\
1 & 1 & n-2
\end{array}\right) .
$$

Therefore, $R E^{D}\left(K_{n}\right)=\frac{3 n-5}{n-1} . \triangleright$

Theorem 4.2. The minimum dominating Randic energy of star graph $K_{1, n-1}$ is

$$
R E^{D}\left(K_{1, n-1}\right)=\sqrt{5} .
$$

$\triangleleft$ Let $K_{1, n-1}$ be the star graph with vertex set $V=\left\{v_{1}, v_{2}, \ldots, v_{n}\right\}$. Here $v_{0}$ be the center. The minimum dominating set $=D=\left\{v_{0}\right\}$. The minimum dominating Randic matrix is

$$
R^{D}\left(K_{1, n-1}\right)=\left[\begin{array}{cccccc}
1 & \frac{1}{\sqrt{n-1}} & \frac{1}{\sqrt{n-1}} & \cdots & \frac{1}{\sqrt{n-1}} & \frac{1}{\sqrt{n-1}} \\
\frac{1}{\sqrt{n-1}} & 0 & 0 & \cdots & 0 & 0 \\
\frac{1}{\sqrt{n-1}} & 0 & 0 & \cdots & 0 & 0 \\
\vdots & \vdots & \vdots & \ddots & \vdots & \vdots \\
\frac{1}{\sqrt{n-1}} & 0 & 0 & \cdots & 0 & 0 \\
\frac{1}{\sqrt{n-1}} & 0 & 0 & \cdots & 0 & 0
\end{array}\right] .
$$

Characteristic equation is

$$
(\lambda)^{n-2}\left[\lambda^{2}-\lambda-1\right]=0
$$




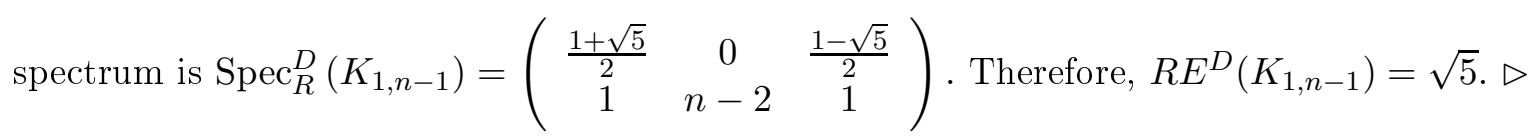

Теорема 4.3. The minimum dominating Randic energy of Crown graph $S_{n}^{0}$ is

$$
R E^{D}\left(S_{n}^{0}\right)=\frac{(4 n-7)+\sqrt{4 n^{2}-8 n+5}}{n-1} .
$$

$\triangleleft$ Let $S_{n}^{0}$ be a crown graph of order $2 n$ with vertex set $\left\{u_{1}, u_{2}, \ldots, u_{n}, v_{1}, v_{2}, \ldots, v_{n}\right\}$ and minimum dominating set $=D=\left\{u_{1}, v_{1}\right\}$. The minimum dominating Randic matrix is

$$
R^{D}\left(S_{n}^{0}\right)=\left[\begin{array}{cccccccccc}
1 & 0 & 0 & \ldots & 0 & 0 & \frac{1}{n-1} & \ldots & \frac{1}{n-1} & \frac{1}{n-1} \\
0 & 0 & 0 & \ldots & 0 & \frac{1}{n-1} & 0 & \ldots & \frac{1}{n-1} & \frac{1}{n-1} \\
0 & 0 & 0 & \ldots & 0 & \frac{1}{n-1} & \ldots & \frac{1}{n-1} & 0 & \frac{1}{n-1} \\
\vdots & \vdots & \vdots & \ddots & \vdots & \vdots & \vdots & \ddots & \vdots & \vdots \\
0 & 0 & 0 & \ldots & 0 & \frac{1}{n-1} & \ldots & \frac{1}{n-1} & \frac{1}{n-1} & 0 \\
0 & \frac{1}{n-1} & \frac{1}{n-1} & \ldots & \frac{1}{n-1} & 1 & 0 & \ldots & 0 & 0 \\
\frac{1}{n-1} & 0 & \frac{1}{n-1} & \ldots & \frac{1}{n-1} & 0 & 0 & \ldots & 0 & 0 \\
\vdots & \vdots & \vdots & \ddots & \vdots & \vdots & \vdots & \ddots & \vdots & \vdots \\
\frac{1}{n-1} & \frac{1}{n-1} & 0 & \ldots & \frac{1}{n-1} & 0 & 0 & \ldots & 0 & 0 \\
\frac{1}{n-1} & \frac{1}{n-1} & \frac{1}{n-1} & \ldots & 0 & 0 & 0 & \ldots & 0 & 0
\end{array}\right] .
$$

Characteristic equation is

$$
\left(\lambda+\frac{1}{n-1}\right)^{n-2}\left(\lambda-\frac{1}{n-1}\right)^{n-2}\left(\lambda^{2}-\frac{1}{n-1} \lambda-1\right)\left(\lambda^{2}-\frac{2 n-3}{n-1} \lambda+\frac{n-3}{n-1}\right)=0
$$

spectrum is

$\operatorname{Spec}_{R}^{D}\left(S_{n}^{0}\right)=\left(\begin{array}{cccccc}\frac{(2 n-3)+\sqrt{4 n-3}}{2(n-1)} & \frac{1+\sqrt{4 n^{2}-8 n+5}}{2(n-1)} & \frac{(2 n-3)-\sqrt{4 n-3}}{2(n-1)} & \frac{1}{n-1} & \frac{-1}{n-1} & \frac{1-\sqrt{4 n^{2}-8 n+5}}{2(n-1)} \\ 1 & 1 & 1 & n-2 & n-2 & 1\end{array}\right)$.

Therefore, $R E^{D}\left(S_{n}^{0}\right)=\frac{(4 n-7)+\sqrt{4 n^{2}-8 n+5}}{n-1} . \triangleright$

Theorem 4.4. The minimum dominating Randic energy of complete bipartite graph $K_{n, n}$ of order $2 n$ with vertex set $\left\{u_{1}, u_{2}, \ldots, u_{n}, v_{1}, v_{2}, \ldots, v_{n}\right\}$ is

$$
R E^{D}\left(K_{n, n}\right)=\frac{2 \sqrt{n-1}}{\sqrt{n}}+2
$$

$\triangleleft$ Let $K_{n, n}$ be the complete bipartite graph of order $2 n$ with vertex set $\left\{u_{1}, u_{2}, \ldots, u_{n}, v_{1}, v_{2}, \ldots, v_{n}\right\}$. The minimum dominating set $=D=\left\{u_{1}, v_{1}\right\}$. The minimum 
dominating Randic matrix is

$$
R^{D}\left(K_{n, n}\right)=\left[\begin{array}{ccccccccc}
1 & 0 & 0 & 0 & \ldots & \frac{1}{n} & \frac{1}{n} & \frac{1}{n} & \frac{1}{n} \\
0 & 0 & 0 & 0 & \ldots & \frac{1}{n} & \frac{1}{n} & \frac{1}{n} & \frac{1}{n} \\
0 & 0 & 0 & 0 & \ldots & \frac{1}{n} & \frac{1}{n} & \frac{1}{n} & \frac{1}{n} \\
0 & 0 & 0 & 0 & \ldots & \frac{1}{n} & \frac{1}{n} & \frac{1}{n} & \frac{1}{n} \\
\vdots & \vdots & \vdots & \vdots & \ddots & \vdots & \vdots & \vdots & \vdots \\
\frac{1}{n} & \frac{1}{n} & \frac{1}{n} & \frac{1}{n} & \ldots & 1 & 0 & 0 & 0 \\
\frac{1}{n} & \frac{1}{n} & \frac{1}{n} & \frac{1}{n} & \ldots & 0 & 0 & 0 & 0 \\
\frac{1}{n} & \frac{1}{n} & \frac{1}{n} & \frac{1}{n} & \ldots & 0 & 0 & 0 & 0 \\
\frac{1}{n} & \frac{1}{n} & \frac{1}{n} & \frac{1}{n} & \ldots & 0 & 0 & 0 & 0
\end{array}\right]
$$

Characteristic equation is

$$
\lambda^{2 n-4}\left(\lambda^{2}-\frac{n-1}{n}\right)\left[\lambda^{2}-2 \lambda+\frac{n-1}{n}\right]=0 .
$$

Hence, $\operatorname{spectrum}$ is $\operatorname{Spec}_{R}^{D}\left(K_{n, n}\right)=\left(\begin{array}{ccccc}1+\sqrt{\frac{1}{n}} & \frac{\sqrt{n-1}}{\sqrt{n}} & 1-\sqrt{\frac{1}{n}} & 0 & -\frac{\sqrt{n-1}}{\sqrt{n}} \\ 1 & 1 & 1 & 2 n-4 & 1\end{array}\right)$. Therefore, $R E^{D}\left(K_{n, n}\right)=\frac{2 \sqrt{n-1}}{\sqrt{n}}+2 . \triangleright$

Definition 4.1. The friendship graph, denoted by $F_{3}^{(n)}$, is the graph obtained by taking $n$ copies of the cycle graph $C_{3}$ with a vertex in common. $V\left(F_{n}\right)=2 n+1$.

Теорема 4.5. The minimum dominating Randic energy of Friendship graph $F_{n}^{3}$ is

$$
R E_{D}\left(F_{n}^{3}\right)=n+1 .
$$

$\triangleleft$ Let $F_{3}^{(n)}$ be the friendship graph with $2 n+1$ vertices. Here $v_{1}$ be the center. The minimum dominating set $=D=\left\{v_{1}\right\}$. The minimum dominating Randic matrix is

$$
R^{D}\left(F_{n}^{3}\right)=\left[\begin{array}{cccccccc}
1 & \frac{1}{2 \sqrt{n}} & \frac{1}{2 \sqrt{n}} & \frac{1}{2 \sqrt{n}} & \frac{1}{2 \sqrt{n}} & \ldots & \frac{1}{2 \sqrt{n}} & \frac{1}{2 \sqrt{n}} \\
\frac{1}{2 \sqrt{n}} & 0 & \frac{1}{2} & 0 & 0 & \ldots & 0 & 0 \\
\frac{1}{2 \sqrt{n}} & \frac{1}{2} & 0 & 0 & 0 & \ldots & 0 & 0 \\
\frac{1}{2 \sqrt{n}} & 0 & 0 & 0 & \frac{1}{2} & \ldots & 0 & 0 \\
\frac{1}{2 \sqrt{n}} & 0 & 0 & \frac{1}{2} & 0 & \ldots & 0 & 0 \\
\vdots & \vdots & \vdots & \vdots & \vdots & \ddots & \vdots & \vdots \\
\frac{1}{2 \sqrt{n}} & 0 & 0 & 0 & 0 & \ldots & 0 & \frac{1}{2} \\
\frac{1}{2 \sqrt{n}} & 0 & 0 & 0 & 0 & \ldots & \frac{1}{2} & 0
\end{array}\right] .
$$

Characteristic equation is

$$
\lambda\left(\lambda+\frac{1}{2}\right)^{n}\left(\lambda-\frac{1}{2}\right)^{n-1}\left(\lambda-\frac{3}{2}\right)=0
$$

Hence, spectrum is

$$
\operatorname{Spec}_{R}^{D}\left(F_{n}^{3}\right)=\left(\begin{array}{cccc}
\frac{3}{2} & \frac{1}{2} & 0 & \frac{-1}{2} \\
1 & n-1 & 1 & n
\end{array}\right) .
$$

Therefore, $R E^{D}\left(F_{n}^{3}\right)=n+1$. $\triangleright$ 
Tеорема 4.6. The minimum dominating Randic energy of Cocktail party graph $K_{n \times 2}$ is

$$
R E^{D}\left(K_{n \times 2}\right)=\frac{4 n-6}{n-1} .
$$

$\triangleleft$ Let $K_{n \times 2}$ be a Cocktail party graph of order $2 n$ with vertex set $\left\{u_{1}, u_{2}, \ldots, u_{n}, v_{1}, v_{2}, \ldots, v_{n}\right\}$. The minimum dominating set $=D=\left\{u_{1}, v_{1}\right\}$. The minimum dominating minimum dominating Randic matrix is

$$
R^{D}\left(K_{n \times 2}\right)=\left[\begin{array}{ccccccccc}
1 & \frac{1}{2 n-2} & \frac{1}{2 n-2} & \frac{1}{2 n-2} & \cdots & 0 & \frac{1}{2 n-2} & \frac{1}{2 n-2} & \frac{1}{2 n-2} \\
\frac{1}{2 n-2} & 0 & \frac{1}{2 n-2} & \frac{1}{2 n-2} & \cdots & \frac{1}{2 n-2} & 0 & \frac{1}{2 n-2} & \frac{1}{2 n-2} \\
\frac{1}{2 n-2} & \frac{1}{2 n-2} & 0 & \frac{1}{2 n-2} & \cdots & \frac{1}{2 n-2} & \frac{1}{2 n-2} & 0 & \frac{1}{2 n-2} \\
\frac{1}{2 n-2} & \frac{1}{2 n-2} & \frac{1}{2 n-2} & 0 & \cdots & \frac{1}{2 n-2} & \frac{1}{2 n-2} & \frac{1}{2 n-2} & 0 \\
\vdots & \vdots & \vdots & \vdots & \ddots & \vdots & \vdots & \vdots & \vdots \\
0 & \frac{1}{2 n-2} & \frac{1}{2 n-2} & \frac{1}{2 n-2} & \cdots & 1 & \frac{1}{2 n-2} & \frac{1}{2 n-2} & \frac{1}{2 n-2} \\
\frac{1}{2 n-2} & 0 & \frac{1}{2 n-2} & \frac{1}{2 n-2} & \cdots & \frac{1}{2 n-2} & 0 & \frac{1}{2 n-2} & \frac{1}{2 n-2} \\
\frac{1}{2 n-2} & \frac{1}{2 n-2} & 0 & \frac{1}{2 n-2} & \cdots & \frac{1}{2 n-2} & \frac{1}{2 n-2} & 0 & \frac{1}{2 n-2} \\
\frac{1}{2 n-2} & \frac{1}{2 n-2} & \frac{1}{2 n-2} & 0 & \cdots & \frac{1}{2 n-2} & \frac{1}{2 n-2} & \frac{1}{2 n-2} & 0
\end{array}\right] .
$$

Characteristic equation is

$$
\lambda^{n-1}\left(\lambda+\frac{1}{n-1}\right)^{n-2}(\lambda-1)\left[\lambda^{2}-\frac{2 n-3}{n-1} \lambda+\frac{n-3}{n-1}\right]=0 .
$$

Hence, spectrum is

$$
\operatorname{Spec}_{R}^{D}\left(K_{n \times 2}\right)=\left(\begin{array}{ccccc}
\frac{2 n-3+\sqrt{4 n-3}}{2(n-1)} & 1 & \frac{2 n-3-\sqrt{4 n-3}}{2(n-1)} & 0 & \frac{-1}{n-1} \\
1 & 1 & 1 & n-1 & n-2
\end{array}\right) .
$$

Therefore, $R E^{D}\left(K_{n \times 2}\right)=\frac{4 n-6}{n-1} . \triangleright$

Acknowledgement. The authors are thankful to the anonymous referee for valuable suggestions and comments for the improvement of the paper. Also, the first author is grateful to Dr. M. N. Channabasappa, Director and Dr. Shivakumaraiah, Principal, Siddaganga Institute of Technology, Tumkur, for their constant support and encouragement.

\section{References}

1. Bozkurt S. B., Gungor A. D., Gutman I., Cevik A. S. Randic matrix and Randic energy // MATCH Commum. Math. Comput. Chem.-2010.-Vol. 64.-P. 239-250.

2. Bozkurt S. B., Gungor A. D., Gutman I. Randic spectral radius and Randic energy // MATCH Commum. Math. Comput. Chem.-2010.-Vol. 64.-P. 321-334.

3. Serife Burcu Bozkurt, Durmus Bozkurt. Sharp Upper Bounds for Energy and Randic Energy // MATCH Commum. Math. Comput. Chem.-2013.-Vol. 70.-P. 669-680.

4. Gutman I., Furtula B., Bozkurt S. B. On Randic energy // Linear Algebra Appl.-2014.-Vol. 442.P. $50-57$.

5. Gutman I. The energy of a graph // Ber. Math. Stat. Sekt. Forschungsz. Graz.-1978.-103.-P. 1-22.

6. Gutman I. The energy of a graph: old and new results // Combinatorics and Applications / A. Betten, A. Khoner, R. Laue and A. Wassermann, eds.-Berlin: Springer, 2001.-P. 196-211.

7. Indulal G., Gutman I., Vijayakumar A. On distance energy of graphs // Match Commun. Math. Comput. Chem.-2008.-Vol. 60.-P. 461-472.

8. Rajesh Kanna M. R., Dharmendra B. N., Sridhara G. Minimum dominating energy of a graph // Int. J. Pure and Appl. Math.-2013.-Vol. 85, № 4.-P. 707-718. 
Received August 6, 2016.

P. Siva Kota Reddy, Prof.

Department of Mathematics

Siddaganga Institute of Technology,

B. H. Road, Tumkuru-572 103, Karnataka, INDIA

E-mail: pskreddy@sit.ac.in; reddy_math@yahoo.com

K. N. Prakasha, Prof.

Department of Mathematics

Vidyavardhaka College of Engineering

P. B. No. 206, Gokulam III Stage, Mysore-570 002, Karnataka, INDIA

E-mail: prakashamaths@gmail.com

V. M. Siddalingaswamy, Prof.

Department of Mathematics

JSS Academy of Technical Education

Uttarahalli-Kengeri Main Road, Bangalore-560 060, INDIA

E-mail: swamy vms@yahoo.com

\section{МИНИМАЛЬНАЯ ДОМИНИРУЮЩАЯ ЭНЕРГИЯ РАНДИЧА ГРАФА}

Сива Кота Редди П., Пракаша К. Н., Сиддалингасвами В. М.

В данной работе мы ввели понятие и вычислили минимальную доминирующую энергию Рандича графа. Кроме того, были найдены верхняя и нижняя границы для минимальной доминирующей энергии Рандича.

Ключевые слова: минимальный доминирующий набор, минимальные доминирующие собственные значения Рандича, минимальная доминирующая энергия Рандича. 